\title{
Der „Medienpapst"
}

\author{
Statements von Gerd Bacher, Hubert \\ Feichtlbauer, Sigmund Gottlieb und Otto B. \\ Roegele
}

"Communicatio Socialis“ hat Medienvertreter um kurze Statements zu den Fragen gebeten, was nach ihrer persönlichen Einschätzung dazu geführt hat, Johannes Paul II. „Medienpapst“ zu nennen:

- was seine Medienausstrahlung begründete,

- zu welchen Wirkungen, Folgen, Veränderungen seine starke und permanente Medienpräsenz geführt hat,

- ob durch die Fokussierung auf die Medienwirkung der Person des Papstes nicht auch strukturelle Probleme und innere Konflikte in der Kirche überdeckt worden sind,

- ob die Medienausstrahlung des Papstes nachhaltige Wirkung für Religion und Gesellschaft zeigt oder nur einen vorübergehenden "Event"-Charakter hatte,

- ob die Bezeichnung „Medienpapst“ das richtige Wort war.

Die Statements dazu stammen von:

Gerd Bacher: Geboren 1925, begann seine journalistische Tätigkeit in Salzburg, setzte sie zunächst in Wien fort, war dann drei Jahre Leiter des Fritz Molden Verlages, ehe er 20 Jahre lang zwischen 1967 und 1994 (mit Unterbrechungen) als Generalintendant den Österreichischen Rundfunk (ORF) leitete.

Hubert Feichtlbauer: Geboren 1932, arbeitete zuerst als Journalist bei Zeitungen in Linz, Salzburg und Wien, bevor er von 1978 bis 1984 Chefredakteur der katholischen Wochenzeitung „Die Furche“ war. Seit 1992 ist er freier Journalist, Mitarbeiter beim ORF und Buchautor; von 1999 bis 2002 war er Vorsitzender der Plattform „Wir sind Kirche“.

Sigmund Gottlieb: Geboren 1951, begann als Redakteur beim „Münchner Merkur", wechselte 1985 zum ZDF und arbeitet seit 1991 beim Bayerischen Rundfunk, wo er seit 1995 Chefredakteur und seit 2001 gleichzeitig stellvertretender Fernsehdirektor ist.

Otto B. Roegele: Geboren 1920, trat 1948 in die Redaktion des „Rheinischen Merkur" ein, war seit 1949 dessen Chefredakteur, seit 1963 Herausgeber. 1963 wurde er ordentlicher Professor der Universität München und Vorstand des Instituts für Kommunikationswissenschaft (Zeitungswissenschaft); von 1967 bis 1988 war er Leiter der Abteilung Kommunikationswissenschaft und Ergänzungsstudium der Hochschule für Fernsehen und Film in München. Am 6. August 2005 vollendete Otto B. Roegele sein 83. Lebensjahr. 


\section{Gerd Bacher:}

Als Johannes Paul II. das erste Mal Deutschland besuchte, begrüßte ihn August Everding mit ungefähr folgenden Worten: Wir danken dem Heiligen Geist, dass er einen Autor und Schauspieler zum Papst ausersehen hat. Everding, selbst ein großer Theater- = Medienmann, wollte damit nicht nur einen verbalen Gag landen, was ihm weltweit gelang, sondern seiner Freude Ausdruck geben, dass der Mann an der Spitze seiner Kirche die allgegenwärtige Mediengesellschaft $z u$ nutzen verstünde. Den Papst belustigte diese Anrede, nichts deutete darauf hin, dass sie ihm zuwider wäre.

Das Prädikat „Medienpapst“ trifft, bei aller zeitgeistigen Oberflächlichkeit, sicherlich eine der ganz großen Wirkmächte dieses Pontifikats. Es gab vor Wojtyla keinen Papst, der die Weltöffentlichkeit und die Weltmedien so $z u$ beschäftigen und $z \mathfrak{u}$ faszinieren vermochte. Jeder seiner Nachfolger wird auch an diesem Beispiel einer nicht nur im religiösen Bereich nie dagewesenen „Öffentlichkeitsarbeit" gemessen werden. Johannes Paul II. beantwortete quasi als Nebenprodukt seiner medialen Mission immer wieder gestellte, aber vordem kaum bean twortete Fragen: Braucht man heute überhaupt noch einen Papst? Ist Rom nicht längst überholt? Sollte der Vatikan nicht einem Kirchenparlament Platz machen? Hat der Papst die von Stalin „bezweifelten“ Divisionen? Ist eine angeblich so zeitgeistferne Institution überhaupt noch im Stande, der Welt etwas zu sagen, gehört zu werden? Gibt es im Vatikan Modernität? Überholte Fragen, angesichts der Präsenz dieses Papstes, wie sie kein Machthaber je hatte. Und noch im Sterben versammelte er Freunde, Gegner und Gleichgültige um sich, zu keinem Anderen wären sie in solcher Zahl und Macht gekommen. Wäre der Preis der Wiedervereinigung der christlichen Kirchen die Aufgabe des Papsttums, so hätte ihn Johannes Paul II. als zu hoch definiert.

Was hat die Medienausstrahlung dieses Papstes begründet? Das ist die unbeantwortete Frage nach dem Wesen von Charisma. Er war die meistgehörte Stimme in einer denkbar lauten, schrillen, ehrfurchtslosen Zeit. Diese Zeit nannte ihn denn auch einen Pop-Super-Star und verstand die Beleidigung als Kompliment. Aber es gibt auch einige ganz praktische Erklärungen. Der Mann aus dem kommunistischen Osten, ein Papst, der wie ein Mann und nicht wie eine Tante aussieht und spricht, die Kraft, die er seinen Polen zum Sieg über den Kommunismus verlieh, das Johnny-Walker-Syndrom, der Mann, der zu allen kam, seine jedermann verständlichen Worte gegen Turbokapitalismus, Armut, Krieg, die er nicht an irgendjemanden, sondern an die Schuldigen richtete, seine Sprachgewalt, die ihn auch und besonders 
noch, als er schon gar keine Sprache mehr hatte, zu einem Heros der Jugend machte.

Die Frage, ob durch die Fokussierung auf die Medienwirkung des Papstes nicht auch strukturelle Probleme und innere Konflikte in der Kirche überdeckt worden sind, beruht auf einem Denkfehler. Überdeckt ist da gar nichts geworden, so gerne es auch viele und besonders viele im Vatikan gehabt hätten. Diese Probleme, die niemand bestreiten kann, sind dem Medienpapst von den Medien vorgeworfen worden, wie keinem vor ihm. Weil eben dieser Papst so interessierte, befasste sich die Öffentlichkeit dermaßen mit „seiner Intransigenz, seiner dogmatischen Verzopftheit, mit seinem Kirchenbild, das der Zeit nicht mehr entspricht". (Je ferner der Heide, desto inniger seine Sorge um die rechte Kirche.) Nichts wurde da überdeckt. Zur Lösung bedarf es eines anderen Papstes, freilich von der Willensstärke Wojtylas. Johannes Paul II. nachhaltige Wirkung hängt von seinen Nachfolgern ab. Er hat ihnen eine Weltkirche überlassen, die wie nie zuvor weltweiten Respekt genießt. Die katholische Krise ist keine Weltkrise, sie heißt Europa. Wer die „innerkirchlichen Fragen“ löst, hat die Medien auf seiner Seite. Irgendein großer Journalist hat den Satz gesagt: „Die Journalisten haben alle schon Gott interviewt, sie wissen nur nicht, wo er wohnt."

Er war der Medienpapst. Aber es möge sich keiner einbilden, dass ihn die Medien, so wie sie es mit vielen Politikern treiben, $z \mathfrak{u}$ instrumentalisieren vermochten. Das war umgekehrt. Er war nie katholischer Schauspieler auf Welttournee.

Ich hatte das Glück einer sehr langen Privataudienz bei Johannes Paul II. Wir redeten viel über Medien und Mediengesellschaft. Er war Fachmann, Gott sei Dank, kein Profi. Zum Schluss sagte er mir: „Der liebe Gott hat mich ausgesucht, weil ich Kraft und Gesundheit zu dieser Wanderung um die Welt habe (da hatte er das lebensgefährdende Attentat bereits hinter sich ), und weil mir die Medien nicht fremd sind. Er will, dass ich die Instrumente nutze."

Das sagte er in seinem schönen, langsamen, gutteralen Deutsch.

\section{Hubert Feichtlbauer:}

Die persönliche Ausstrahlung von Johannes Paul II. war groß, und sie wurde auch über die Medien spürbar. Er sprach anders als Politiker, anders aber auch als Popstars, redete niemandem nach dem Mund, verhieß keine Bequemlichkeiten, machte überhaupt keine Versprechungen und bestach auch durch großartige Inszenierungen. In einer Zeit, die mehr nach Design als nach Sein fragt, wirkte die hehre weiße Gestalt im 
Papamobil unter Menschenmassen oder auf entrückter Schaubühne bei Eucharistiefeiern geradezu außerirdisch. Trotzdem würde ein Mensch ohne persönliches Charisma auch in solcher Aufmachung nicht eine derart starke Wirkung entfalten. Das Entscheidende bei Johannes Paul II. war schon seine Echtheit oder „Authentizität“, denn er bot neben Design auch Sein: eine uneinholbare Mischung. Ihm glaubte man, was er verkündete, und verband seine Botschaft nicht mit hohlem Phrasengedresch oder "Wein predigen und Wasser trinken“. Freilich: Nichts sprach dafür, dass seine Zuhörerinnen und Zuhörer auch befolgten, was er ihnen auftrug.

Seine Hauptwirkung war wohl, die katholische Kirche in der säkularen Welt präsent zu halten und wichtige Positionen in Fragen der Individualund Sozialethik bekannt zu machen. Die Verhältnismäßigkeit der Wiedergabe seiner Ausführungen wurde freilich nie gewahrt. Auch wenn man uns heute hundertmal vorrechnet, dass seine Texte zur Sexualität nur rund fünf Prozent aller seiner Aussagen ausmachten - auch bei der 104. Auslandsreise wurde wieder nur berichtet, er habe Empfängnisregelung und Abtreibung verdammt. Und allzu oft tat er es ja in der Tat in ein und demselben Satz. Das geht bei Benedikt XVI. schon wieder genau so weiter. Da muss man schon mit Insiderohren hinhören, um mitzukriegen, dass der Nachfolger beim ersten Mal die Verhütung nicht als Zwillingsthema behandelte. Sehr positiv war, dass auch das unzweideutige Nein Johannes Pauls zu Kriegen und Gewaltakten aller Art in aller Welt gehört und ebenso wie sein Eintreten für eine gerechte Welt- und Gesellschaftsordnung begrüßt wurde. Allen Aufrufen zur Bekämpfung von Armut und Elend wurde freilich das völlig irreale Kondomverbot entgegengehalten, durch das er selbst die globale Wirkung seiner Moralappelle schmälerte. Innerhalb seiner Kirche hat Johannes Paul sicher Millionen Menschen vor allem in Elendsländern allein durch seine Besuche Mut gemacht und Hoffnung vermittelt, aber die Gewissenslast des Verbots so genannter künstlicher Verhütungsmittel hat er nicht von ihnen genommen. Personen im kirchlichen Kernsektor hat diese Widersprüchlichkeit immer wieder gestört. Interessierten Beobachtern am ekklesialen Rand, die sich nie um diese Obsession gekümmert haben, gefiel er umso besser. Reevangelisiert (früher hätte man gesagt: bekehrt) hat der Papst Europa nicht. Aber damit wäre ein Einzelner, selbst wenn er übermenschliche Qualitäten hätte, überfordert. Freilich musste man bisweilen schon den Eindruck bekommen, dass der Papst nicht ungern mit solchen Qualitäten identifiziert worden wäre.

Wurden Strukturprobleme in der katholischen Kirche durch diese massive Medienpräsenz des Papstes überdeckt? Ja, sicher. Alles, was man als Strukturproblem abqualifizieren konnte, wurde minimalisiert: 
Nur Kleingeister streiten über Priesterehen und Frauenweihen, statt diesem großartigen Papst zu folgen! Als ob der Rang der Frau in einer Kirche, die als Anwältin von Menschenrechten und Menschenwürde alle Welt belehrt, eine Strukturfrage wäre! Noch schlimmer ist, dass für viele überhaupt der Eindruck entstehen musste, der Papst verkörpere in seinem Amt und damit auch in seiner Person alles, was katholisch ist. Tragischer Umkehrschluss: Wer in manchen Fragen, die nicht zum Glaubenskern gehören, anders denkt als der Papst, ist nicht (mehr) katholisch. Das ist schlechte Theologie und widerspricht aller immer wieder beteuerten ökumenischen Gesinnung. Wenn die katholische Kirche die Zielsetzung einer Christeneinheit in versöhnter Vielfalt ernst nimmt und ihr Amtsverständnis an biblischen Ursprüngen und historischen Lernprozessen misst, muss ein Papst sich künftig zurücknehmen. Es würde seine Autorität mehr stärken als tägliche Menschheitsbelehrung.

Die Medienwirkung von Johannes Paul II. hatte ihre positiven und ihre negativen Seiten. Sie hat einfache Gläubige gestärkt und kritisch mitdenkende verstört. Ehrlich sei gesagt: Das Erste ist wichtiger. Aber das Zweite wäre nicht notwendig. Ein Papst könnte auch einen noch stärker überzeugenden, widerspruchsfreien, barmherzigen Glauben ausstrahlen, der Demut nicht nur verbal, sondern auch in der Körpersprache ausdrückt.

War „Medienpapst“ das richtige Wort für Johannes Paul? Die Frage ist müßig. Solche Wörter entstehen - meist ohnehin in Medien - ohne vorherige Konsultation von Wissenschaftern und Wörterbüchern. Und sie bleiben, wenn alle verstehen, was damit gemeint ist. Bei Johannes Paul hat man es gewusst.

\section{Sigmund Gottlieb:}

Das Zauberwort heißt Präsenz. Diese Präsenz lebte fort über den Tod hinaus. Aufgebahrt im Petersdom umgab Johannes Paul II. noch diese Präsenz. Das Da-Sein, das Unter-den-Menschen-Sein, das Für-dieMenschen-Dasein. Diesem Eindruck konnte sich keiner der Pilger entziehen.

So blieb Karol Wojtyla selbst im Tod ein Medienstar: Nie zuvor in der Geschichte des Papsttums gab es eine stärkere Präsenz. Nie zuvor waren die Kameraaugen näher am Leichnam eines Papstes, wie er in seinem roten Pontifikalgewand aufgebahrt lag. In dem Vierteljahrhundert seines Pontifikats wufde jeder Auftritt zu einem außergewöhnlichen Medienereignis. Das lag einmal daran, dass dieser Papst aus dem Holz geschnitzt war, aus dem Mega-Kommunikatoren gemacht sind: glänzend ausse- 
hend, sportlich, ein Schauspiel-Talent (schließlich war er ja Schauspieler in seiner Jugend), vielsprachig und wieder diese Präsenz, wo immer er auftrat.

Und er war sich der Wirkung der modernen Massenmedien bewusst. Er wusste um ihre Bedeutung, und deshalb ließ er keinen Flirt mit der Kamera aus. Johannes Paul II. wusste um die kommunikative Macht der Bilder. So kam es, dass er das Schweigen des Vatikans brach und die katholische Kirche stattdessen zum Sprechen brachte. Neue Zeiten für die Vatikan-Journalisten: Auf einmal wurden Interviews an Bord des Papst-Flugzeugs möglich. Dies hatte es nie zuvor gegeben. Er wusste die Symbolkraft und die verstärkende Wirkung der Fernsehbilder zu nutzen: zum Beispiel während seiner Israel-Reise im Jahr 2000, als er an der Klagemauer nach jüdischer Tradition einen Gebetszettel in einen Mauerspalt steckte.

Nie war dieser Papst wohl eine so öffentliche Person wie in seinem körperlichen Leiden und Sterben, das bei jedem anderen die Zeitspanne der größten Privatheit ist. Nie konnte das Leiden Christi so präsent gemacht werden wie in dieser Zeit. Leiden und Sterben als eine öffentliche Angelegenheit.

Wie sehr dieser Papst nicht nur die Menschen in aller Welt, sondern auch uns Journalisten beschäftigt hat, zeigt eine unglaubliche Zahl. Die Betreiber einer speziellen Internet-Suchmaschine, sie heißt „Global Languages“, haben ermittelt, dass in den ersten 24 Stunden nach dem Tod des Papstes 35000 Artikel über ihn verfasst wurden, zehnmal soviel wie nach der Wiederwahl des amerikanischen Präsidenten.

Wie stark und wie nachhaltig war die Wirkung Johannes Pauls II.? Was war das Besondere, das Geheimnisvolle seiner Wirkung, seiner Präsenz? Ich bin überzeugt: Selbst die ganz herausragenden kommunikativen Fähigkeiten dieses Papstes haben ihre volle Wirkung nur vor dem Hintergrund mehrerer besonderer historischer Situationen entfalten können: Globalisierung, Zusammenbruch des Kommunismus, wachsende Kriegsgefahr weltweit. Angesichts dieser dramatischen Veränderungen, in die sich dieser politische Papst mit dem ihm eigenen Engagement immer wieder einmischte, suchten zig Millionen Menschen auf diesem Globus Halt, Orientierung, Ver-Gewisserung, Ver-Sicherung, Vor-Bild. Diese Suche fand weit über die Grenzen des Katholizismus hinaus statt. Auch Atheisten erwarteten sich von Karol Wojtyla eine Antwort. In den Tagen vor den Beisetzungsfeierlichkeiten und vor der Amtseinführung Benedikts XVI. traf ich in Rom auf Jugendliche aus der ganzen Welt. Alle Gespräche, die ich führen konnte, lassen sich zu einer Kernaussage zusammenfassen: Johannes Paul II. hatte einen klaren Standpunkt. Eine Haltung, die auf einem festen Wertefundament gründet. Haltung, die sich 
nicht verbiegt und verbeugt vor dem Zeitgeist. Haltung, die sich den Beliebigkeiten unserer westlichen Spaßgesellschaften entgegenstellt.

Die Kritik, innerkirchliche Kontroversen als Thema sei im Rahmen der ausführlichen Papst-Berichterstattung zu kurz gekommen, kann ich nur zu einem geringen Teil nachvollziehen. Auch dieses Thema wurde in den zahlreichen Gesprächsanalysen immer wieder abgehandelt. Allerdings ist genauso deutlich darauf hinzuweisen, dass diese Problematik in jenen Tagen nicht im Mittelpunkt stehen konnte und sollte. Neben der dramaturgischen und emotionalen Wucht dieses Weltereignisses trat natürlich auch ihre politische Dimension. Vergessen wir doch nicht, dass etwa das Requiem für den verstorbenen Papst auch ein weltpolitisches Ereignis war. An seinem Sarg hatten sich mehr als 200 Spitzenpolitiker aus aller Welt versammelt. Damit war es die größte Ansammlung von Spitzenpolitikern in der Geschichte und ein Beweis dafür, wie stark dieser Papst Einfluss auf die Weltpolitik genommen hat.

\section{Otto B. Roegele:}

Es war Karl Forster, der als erster Autor auf die Öffentlichkeitswirkung von Johannes Paul II. auch in Deutschland hinwies. Um die Ursachen des überraschenden Vorgangs zu klären, nutzte er Ergebnisse von Untersuchungen des Instituts für Demoskopie Allensbach, die nach klassischem Verfahren in Vorher- und Nachher-Befragungen gewonnen worden waren. Der Befund war eindeutig:

Vor dem Papstbesuch hatte eine erhebliche Mehrheit erklärt, sie erwarte nichts Positives von einem Besuch dieses Papstes in Deutschland; man sollte das dafür vorgesehene Geld lieber für andere Zwecke ausgeben. In der jüngeren Generation war die Ablehnung am stärksten.

Nach dem Papstbesuch im Jahr 1980 ergab die Befragung eine entschiedene Wendung zum Positiven: Die negativen Stimmen waren fast völlig verschwunden; nur noch drei Prozent der 19- bis 20-Jährigen deutschen Katholiken fanden es "nicht gut, dass der Papst diesen Besuch gemacht hat" (Karl Forster: „Popularität nicht durch Zugeständnisse. Allensbacher Zahlen zum Papstbesuch" in "Rheinischer Merkur" vom 8.5.1981, S. 22).

Die positive Einschätzung wurde zu einer Konstanten in der öffentlichen Debatte; Kritiker gerieten zunehmend unter Begründungszwang, wenn sie ihr widersprachen. Auch viele Zeitgenossen, die „mit dem Papst in Rom nichts "am Hut haben" wollten, hörten seiner Stimme mit wachsendem Interesse zu. Das Fernsehen berücksichtigte in Nachrichten und Berichten immer häufiger die großen kirchlichen Ereignisse in Rom und 
die Auslandsreisen des Papstes. Mit dieser Entwicklung beschäftigte sich auch die Bonner Forschungsgruppe „Medien Tenor“. In ihrem Bericht Nr. 137 vom Oktober 2003 gibt es Hinweise auf einige Ergebnisse ihrer Analysen. Sie stehen unter der Überschrift „Johannes Paul Medienstar".

Aber warum trieben die Massenmedien soviel mehr unternehmerischen, journalistischen und technischen Aufwand für diesen Sektor ihrer Programme? Hat etwa das Publikum einen höheren Bedarf angemeldet? Haben die Leute vom Fernsehen selbst herausgefunden, wie vortrefflich die großen liturgischen Manifestationen geeignet sind, ihre Tag- und Nachtprogramme zu füllen?

Jedenfalls trafen sie bei dem Papst aus Polen auf einen ebenso sachverständigen wie (im doppelten Sinne des Wortes) aufnahmebereiten Gesprächspartner. Der gelernte Schauspieler und erfolgreiche Stückeschreiber aus der Krakauer Studentenszene erkannte sogleich die neuen Chancen, die sich hier boten. War es ihm nicht schon gelungen, aus dem antiken Brauch des Kniefalls beim ersten Betreten eines fremden Landes den Auftritt des Staatsgastes zu machen, der Besitz ergreift von den Seelen der Bewohner des Landes, das er erstmals besucht?

\section{Die Öffnung der Isolierzelle}

Mehr als hundert Auslandsreisen zu kirchlichen Jubiläen, Kongressen und Wallfahrtsorten unternahm der Papst in Sondermaschinen der Alitalia, wo er die für den Prominenten und dessen nächste Umgebung vorgesehene abgeschlossene Kabine benutzte. Die begleitenden Journalisten waren im übrigen Teil des Flugzeugs untergebracht.

Eines Tages gelang es einem der „Vaticanisti“, wie die beim Heiligen Stuhl akkreditierten Korrespondenten genannt werden, den vorbeigehenden Papst anzusprechen und ihm eine Frage zum Programm zu stellen. Der Papst ließ sich in einen kleinen Dialog verwickeln, er setzte sich für einen Augenblick unter die Fragensteller und blieb bis zur Ankunft bei ihnen. Er hatte auch einiges Wichtige erfahren können und fand die Begegnung mit den Journalisten ungemein anregend. Die Isolierzelle wurde geöffnet, die Zahl der Journalisten, die sich an den Reisen beteiligten, nahm zu; es mussten mehrere Pools gebildet werden, die abwechselnd in der Papstmaschine mitreisen durften. So erwies sich die Erfüllung der Journalisten-Bitte als eine der besten Eingebungen, die zur Wirkung der päpstlichen Bemühungen beitrugen, ganz abgesehen von dem Prestige-Zuwachs für den Berufszweig der Vatikan-Berichterstatter. 
Im Dienst der „neuen Heiligen“

Eine weitere Neuerung, die sich als sehr wirksam erwies, resultierte aus dem Wunsch des Papstes, die „neuen Heiligen“, ihre Lebensgeschichte und ihre Botschaft für die Menschen in der Welt bekannt zu machen. Er hatte erfahren, dass dort, wo diese neuen Heiligen gelebt und gewirkt hatten, eine besondere Bereitschaft zur Aufnahme dieser Botschaft bestand. Er wollte in eigener Person daran mitwirken.

Dabei traute er sich zu, auf Dolmetscher weithin verzichten zu können. Ein nicht ganz korrekter Ausdruck, so wusste er, konnte sich dem Gedächtnis tiefer einprägen als die polierte Version eines Übersetzers. Dieses Modell direkter Verkündigung durch den Papst setzte freilich eine genaue Planung, gründliche Vorarbeit und tadelloses Zusammenspiel aller Beteiligten voraus, mit dem unerlässlichen Protokoll und (besonders seit dem Attentat auf dem Petersplatz) auch mit den Sicherheitsdiensten.

Dies gilt für die wissenschaftliche und organisatorische Arbeit, die erforderlich ist, um die kirchenrechtlichen Voraussetzungen für eine Kanonisation zu erfüllen, in besonderem Maße. Eine eigene Kongregation, mit internationalen Spezialisten besetzt, ist damit befasst, die Berichte und Zeugnisse über Leben und Wirken der „Kandidaten“ nach strengen Maßstäben zu prüfen, vor allem die Berichte über Wunder, die auf Anrufung des „neuen Heiligen“ geschehen seien, sorgfältig kritisch zu studieren, Zweifel rückhaltlos aufzuklären und sich auch durch die Voten der jeweiligen Heimatdiözesen nicht von diesem Wege abbringen zu lassen.

Johannes Paul II. hat an entscheidenden Sitzungen der Kongregation für die Heiligsprechungen teilgenommen. Er hat es sich selbst nicht leicht gemacht, dem hohen Anspruch gerecht $z u$ werden, den seine Verkündigung erhob. Seine Zuhörer haben auch im Riesenrund des Münchner Stadions gespürt, dass da einer zu ihnen sprach, der mit Kopf und Herz dabei war - eben „authentisch“, wie einige sich ausdrückten, die ihm dort begegnet sind.

Eine ähnliche Sprach-Not erlebten auch Autoren mit hagiographischen Absichten. Um die enormen Anstrengungen der letzten Lebensjahre des Papstes zu charakterisieren, hat man von "Selbstaufopferung“ gesprochen. Wo er keine Worte mehr formen konnte, sollte der Anblick seines Leidens Zeugnis ablegen. „Selbsthingabe“ wäre wohl das Wort der klassischen Hagiographie. Unsere härteren „Zeitgeister“ zogen das Wort „Selbstvermarktung“ vor. Vielleicht ist „Totalhingabe“ geeigneter, um auch die unerhbirte Souveränität dieses Mannes in seinem Umgang mit sich und der Welt zu benennen. Ein Rätsel ist freilich geblieben, was dieses Wort „authentisch“ eigentlich sagen soll. Dass es die Abwehr 
weiteren Nachforschens bedeutet, ist klar. Aber das kann nicht alles sein. Es öffnet nicht den Zugang zu dem Paradox, dass ausgerechnet diese Jugend, die so stark von Schönheit, Erfolg, Show-Effekten und enthemmenden Mitteln angezogen wird, zu diesem Papst hindrängt, um ihn jubelnd zu begrüßen. Sie dankten für seinen Segen, sie spürten sein Wohlwollen.

Wer mit dem Handwerkszeug des Psychologen weiter in sie dringen wollte, kam nicht recht vom Fleck. Auch was der Papst selbst über die Begegnungen mit ihnen dachte, blieb letztlich offen. Wie er das Wort „authentisch" gedeutet hat, verriet er nicht. Es konnte ihm ja nicht verborgen bleiben, dass der laute Dank für seinen Segen etwas anderes war als Annahme seiner Botschaft und Befolgung seines Rates ...

\section{Die Macht des sterbenden Papstes}

Welche Macht dieser Papst über die Menschen ausübte, wurde vollends sichtbar, als er im Sterben lag. In ganz Italien, in Polen, auch in anderen europäischen Ländern setzten sich Millionen Menschen in Bewegung, um von Johannes Paul II. Abschied zu nehmen. Alles geschah unorganisiert, spontan, ohne große öffentliche Debatte, ohne kirchenamtliche Aufforderung. Im Gegenteil: Je deutlicher es wurde, welche Schwierigkeiten mit Unterbringung und Versorgung so vieler Menschen verbunden sein mussten, je dramatischer die Warnungen der amtlichen Stellen formuliert wurden, umso weniger zeigten sich die Menschen, die sich zur Rom-Fahrt entschlossen hatten, beeindruckt. Sie waren nicht aufzuhalten, sie reihten sich einfach ein in die schon kilometerlangen Warteschlangen, richteten sich mit Decken und Mänteln auf zwei bis drei Tage und Nächte unter freiem Himmel ein, besorgten das Nötigste an Nahrung und überließen sich der Hoffnung, dass der Tote, der auf sie wartete, dafür sorgen werde, dass sie von ihm noch Abschied nehmen könnten.

Die Behörden sahen schließlich ein, dass hier nichts mehr $z u$ verbieten war, und verlegten sich auf die Beobachtung einer friedlichen Straßenbesetzung, halfen in Notfällen und waren mit dieser Strategie erfolgreich. So unglaublich es klingen mag: Die Polizeiberichte von Rom zeigen kaum eine frühere Woche, in der es so wenig Straftaten, Überfälle, Diebstähle und ähnliche Übeltaten gab wie in dieser. Es war, nach der Meinung römischer Stadthistoriker, das erste offentliche Wunder des toten Papstes. Als dann auch das Requiem auf dem Petersplatz in Anwesenheit von mehr als zweihundert Regierungsdelegationen mit Königen und Präsidenten aus den unterschiedlichsten Lagern ohne Zwischenfall überstanden war, verstummten die letzten Zweifler. 